\title{
SOME CONSEQUENCES OF THE THEOREMS OF GROSS AND IVERSEN
}

\section{A. J. LOHWATER}

1. Introduction. For the most part, this paper is of a survey character, although we present some relatively little-known results in Section 4. It is our intention to show the extent to which the results of Gross [9], [10] and Iversen [14] have played an important role in the development of several areas in the modern theory of functions. We assume that the reader is familiar with the notation for the cluster set $C\left(f, z_{0}\right)$, the range set $R\left(f, z_{0}\right)$, and the asymptotic set $\Gamma\left(f, z_{0}\right)$ as defined in the introduction to the book [2].

Both Gross and Iversen began with the famous theorem of Picard, that if $z_{0}$ is an isolated point of the set $E \subset D$, and if $f(z)$ is meromorphic in $D-E$, where $E$ is the set of essential singularities of $f(z)$, then the complement $\mathbf{C} R\left(f, z_{0}\right)$ of $R\left(f, z_{0}\right)$ consists of at most two points. If there actually exist points in $\mathbf{C} R\left(f, z_{0}\right)$, they are called Picard-exceptional points, and the first main result of Iversen ([14]; see also [2], Theorem 1.6) asserts that such exceptional points are asymptotic values of $z_{0}$ :

The or e m 1. If $z_{0}$ is an isolated essential singularity of $f(z)$, then $\mathbf{c} R\left(f, z_{0}\right) \subset \Gamma\left(f, z_{0}\right)$.

We remark that, if $z_{0}$ is not an isolated singularity of $f(z)$, but is a limit point of isolated singularities, then again $\mathbf{C} R\left(f, z_{0}\right)$ contains at most two points and

$$
\text { C } R\left(f, z_{0}\right) \subset \bigcap_{z \in N \cap \cap_{E-z_{0}}} \Gamma(f, z),
$$

where $N$ is any neighbourhood of $z_{0}$.

One of the major developments based upon the investigations of Gross and Iversen on the theorem of Picard has been a description of the behaviour of a meromorphic function in the vicinity of a non-isolated set of singularities, and one of the first important results in this direction was the following theorem of af Hällström [11].

$\mathrm{Th}$ e or e m 2. If the set $E$ of essential singularities of a meromorphic 
function $f(z)$ is of capacity zero, then $\mathbf{C} R\left(f, z_{0}\right)$ is of capacity zero for all $z_{0} \in E$.

Cartwright [1] has given the analogue of Theorem 1 for Theorem 2 by showing, under the hypotheses of Theorem 2, that for any $\alpha \in \mathbf{C} R\left(f, z_{0}\right)$, $z_{0} \in E$, either $\alpha \in \Gamma\left(f, z_{0}\right)$ or $\alpha \in \Gamma\left(f, z_{n}\right)$ for every $z_{n}$ in a sequence of points $\left\{z_{n}\right\} \subset E$ such that $\lim _{n \rightarrow \infty} z_{n}=z_{0}$. It should also be remarked that the cluster set $C\left(f, z_{0}\right)$ consists of the extended complex plane (or the Riemann sphere) as the set of singularities $E$ "grows" from a relatively thin set to a set of linear measure zero [2]. However, if, in the above theorems, we relax the requirement that $E$ be of linear measure zero, and, in particular, the set $E$ be allowed to contain a continuum, then $C\left(f, z_{0}\right)$ need not be the Riemann sphere and the foregoing theorems may fail. Thus we find ourselves in a different situation requiring a separate study, where the characteristic problems are those of the boundary behaviour of functions defined in a (simply connected) domain whose boundary consists of more than one point, that is, a domain conformally equivalent to the unit disc.

To state the deeper theorems of Gross and Iversen, we define the boundary cluster set $C_{B}\left(f, e^{i \vartheta_{0}}\right)$ of a function $f(z)$, meromorphic in $|z|<1$, at the point $e^{i \vartheta_{0}}$. We first form the set

$$
C\left(f, 0<\left|\vartheta-\vartheta_{0}\right|<\eta\right)=\bigcup_{0<\left|\vartheta-\vartheta_{0}\right|<\eta} C\left(f, e^{i \vartheta}\right)
$$

for a fixed $\eta>0$, and then define $C_{B}\left(f, e^{i \vartheta_{0}}\right)$ to be the intersection

$$
C_{B}\left(f, e^{i \vartheta_{0}}\right)=\bigcap_{\eta>0} \bar{C}\left(f, 0<\left|\vartheta-\vartheta_{0}\right|<\eta\right)
$$

where $\bar{C}$ is the closure of $C$. Clearly $C_{B}\left(f, e^{i \vartheta_{0}}\right)$ is a closed subset of $C\left(f, e^{i \vartheta_{0}}\right)$, but it need not be connected (cf. [2], p. 81). A second result of Iversen ([15], [16]) may now be stated in terms of cluster sets.

Th e o r e m 3. If $f(z)$ is meromorphic in $|z|<1$, then, at every point $e^{i \vartheta}$ of $|z|=\mathrm{I}$,

$$
\partial C\left(f, e^{i \vartheta}\right) \subseteq C_{B}\left(f, e^{i \vartheta}\right)
$$

where $\partial C\left(f, e^{i \vartheta}\right)$ denotes the frontier of $C\left(f, e^{i \vartheta}\right)$.

Since $C_{B}\left(f, e^{i \vartheta}\right)$ is a subset of $C\left(f, e^{i \vartheta}\right)$, the inclusion (3) is equivalent to saying that $C\left(f, e^{i \vartheta}\right)-C_{B}\left(f, e^{i \vartheta}\right)$ is an open set.

Using the terminology and notation that we have developed in this introduction, we may now state the principal result of Gross and Iversen which has proved to be so fruitful. 
Th e o r e m 4. If $f(z)$ is meromorphic in $|z|<1$, then for each point $e^{i \vartheta}$ on $|z|=1$, every value of $C\left(f, e^{i \vartheta}\right)-C_{B}\left(f, e^{i \vartheta}\right)$ is assumed infinitely often in every neighbourhood of $e^{i \vartheta}$ with two possible exceptions. Furthermore, every such exceptional value is an asymptotic value of $f(z)$ at $e^{i \vartheta}$.

We remark that Theorem 4 is valid whether or not $e^{i \vartheta}$ is a singularity of $f(z)$. The motivation for studying extended versions of Theorem 4 stems from the observation that, whenever $e^{i \vartheta}$ is not an isolated singularity of $f(z)$, the subset $C_{B}\left(f, e^{i \vartheta}\right)$ of $C\left(f, e^{i \vartheta}\right)$ may actually coincide with $C\left(f, e^{i \vartheta}\right)$ so that the open set $C\left(f, e^{i \vartheta}\right)-C_{B}\left(f, e^{i \vartheta}\right)$ is empty. We exhibit an example of a function showing that $C\left(f, e^{i \vartheta}\right)-C_{B}\left(f, e^{i \vartheta}\right)$ may be empty for all $e^{i \vartheta}$ on $|z|=1$; this example, which is a relatively simple Blaschke product, will be useful in the sequel.

Example. Let $w=b(z)$ be a Blaschke product such that the circle $K:|z|=1$ is the derived set, or set of all limit points, of the zeros $\left\{\alpha_{k}\right\}_{k=1}^{\infty}$ of $b(z)$. Then, for each point $e^{i \vartheta}$ on $K$, there is a subsequence $a_{k_{j}}$ of $\left\{a_{k}\right\}$ such that $\lim _{j \rightarrow \infty} a_{k_{j}}=e^{i \vartheta}$. Hence the cluster set $C\left(b, e^{i \vartheta}\right)$ is the closed disc $|w| \leqq 1$. Thus the set (1) is the closed disc $|w| \leqq 1$ for any $\eta>0$, so that $C_{B}\left(b, e^{i \vartheta}\right)$ in (2) is also the closed disc $|w| \leqq 1$ for every $e^{i \vartheta}$ on $K$. It follows that $C\left(b, e^{i \vartheta}\right)-C_{B}\left(b, e^{i \vartheta}\right)$ is the empty set for every $e^{i \vartheta}$ on $K$.

2. Special cluster sets and exceptional values. As we saw at the end of Section 1, the usefulness of Theorem 4 is limited to the case when the singularities $e^{i \vartheta}$ on $K:|z|=1$ are isolated in some sense. Since the set $C\left(f, e^{i \vartheta}\right)-C_{B}\left(f, e^{i \vartheta}\right)$ in Theorem 4 is crucial, as the Example at the end of Section 1 shows, any effort to improve Theorem 4 must be based on sharpening Theorem 3. Let $C_{\text {rad }}\left(f, e^{i \vartheta}\right)$ denote the cluster set of $f(z)$ taken along the radius drawn to $e^{i \vartheta}$, that is, the set of all limiting values of $f(z)$ when the defining sequences are restricted to that radius. We form the set

$$
C_{\text {rad }}\left(f, 0<\left|\vartheta-\vartheta_{0}\right|<\eta ; E\right)=\underset{\substack{0<\left|\vartheta-\vartheta_{0}\right|<\eta \\ e^{i \vartheta} \in K-E}}{\bigcup} C_{\text {rad }}\left(f, e^{i \vartheta}\right),
$$

where $E$ is a given set on $K:|z|=1$, and where $\eta>0$ is fixed. We then define the radial boundary cluster set modulo $E$ at $e^{i \vartheta_{0}}$ to be the intersection

$$
C_{R-E}\left(f, e^{i \vartheta_{0}}\right)=\bigcap_{\eta>0} \bar{C}_{\mathrm{rad}}\left(f, 0<\left|\vartheta-\vartheta_{0}\right|<\eta ; E\right) .
$$


The following theorem, with a restriction on the set $E$, generalizes Theorem 3.

Theorem 5. If $f(z)$ is meromorphic in $|z|<1$, and if $E$ is a set of measure zero on $K$, then, at every point $e^{i \vartheta} \in K$,

$$
\partial C\left(f, e^{i \vartheta}\right) \subseteq C_{R-E}\left(f, e^{i \vartheta}\right)
$$

The example of the Blaschke product $b(z)$ given at the end of Section 1 both illustrates Theorem 5 and provides the motivation for our generalization of Theorem 4. As we have remarked, the open set $C\left(f, e^{i \vartheta}\right)-$ $C_{B}\left(f, e^{i \vartheta}\right)$ is empty for every $e^{i \vartheta} \in K$. However, since $b(z)$ is analytic and bounded in $|z|<1$, it follows from the fact that $b(z)$ is a Blaschke product that $b(z)$ possesses radial limits of modulus 1 almost everywhere on $K$; hence, if we choose $E$ to be that set (of measure zero) on $K$ at which $b(z)$ either possesses no radial limits, or, if radial limits exist, they are of modulus less than 1 , then the set (5) consists of a set lying on $|w|=1$. Since, by Theorem 5 , the set $C\left(b, e^{i \vartheta}\right)-C_{R-E}\left(b, e^{i \vartheta}\right)$ is open, it must be the set $\{|w|<1\}$. However, the exceptional set in Theorem 4, namely a set consisting of at most two points, is larger, as simple examples show (see [2], Chapter 5, for further discussion); the main result in this area is the following theorem.

Theorem 6. If $f(z)$ is meromorphic in $|z|<1$, and if $E$ is an arbitrary set of measure zero on $|z|=1$, then every value of $C\left(f, e^{i \vartheta}\right)$ $C_{R-E}\left(f, e^{i \vartheta}\right)$ is assumed by $f(z)$ in any neighbourhood of $e^{i \vartheta}$, with the possible exception of a set of capacity zero. Furthermore, every exceptional value of $f(z)$ in $C\left(f, e^{i \vartheta}\right)-C_{R-E}\left(f, e^{i \vartheta}\right)$ is an asymptotic value of $f(z)$, either at $e^{i \vartheta}$, or else at a sequence of points on $|z|=1$ having $e^{i \vartheta}$ as a limit point.

An example [2] shows that the exceptional set can actually consist of a given closed set of capacity zero. If the set $E$ is of capacity zero, and consequently of measure zero, it can be shown ([2], Theorem 5.15) that the exceptional set in Theorem 6 is at most countable. An interesting extension of Theorem 6 has been given by Woolf [25], [26]:

The or em 7. If, under the hypotheses of Theorem 6 , the set $E$ is of capacity zero, then $f(z)$ assumes every value $C\left(f, e^{i \vartheta}\right)-C_{R-E}\left(f, e^{i \vartheta}\right)$ in any neighbourhood of $e^{i \vartheta}$, with the possible exception of two values in each component of $C\left(f, e^{i \vartheta}\right)-C_{R-E}\left(f, e^{i \vartheta}\right)$.

We conclude this section with several remarks. First, the importance of the theorems of Gross and Iversen in the modern theory of functions was first pointed out by Doob [4], who gave simple proofs and a fresh reformulation of Theorems 3 and 4, and who gave the so-called "one-sided" 
version [5] of Theorem 3. For a partial description of Doob's generalizations of the results of this section by probabilistic methods, see [18, Chapter 6].

Second, it follows from the investigations of Collingwood, who showed the significance of the notion of Baire category in the theory of boundary behaviour, that for all $e^{i \vartheta}$ on $|z|=1$, except for a countable set, the set $C\left(f, e^{i \vartheta}\right)-C_{B}\left(f, e^{i \vartheta}\right)$ is empty, while, except for a set of first category, the set $C\left(f, e^{i \vartheta}\right)-C_{R-E}\left(f, e^{i \vartheta}\right)$ is empty unless $E$ is residual on $|z|=1$ (cf. [2], Theorem 5.17).

3. Banach-algebraic methods. In this section, we shall deal with certain questions in one of the most fruitful areas of investigation, the theory of Banach algebras of bounded analytic functions, but we shall restrict ourselves to problems related to, or suggested by, the theorems mentioned above. In particular, we shall indicate, in yet another way, how the extension given by Theorem 6 is best possible. (For a full account of the general theory up to ten or twelve years ago, the reader should see Naimark [19], Gelfand, Raikov and Shilov [8], Rickart [20], and Hoffman [12]; Duren's book [6] contains an excellent account of the applications to $H^{p}$ spaces, and Rudin [21] contains material on the theory of functions of several complex variables. Wermer [24] has written one of the best elementary surveys, and [18] contains a chapter which is oriented towards the present point of view.)

Our plan of this section is, of necessity, fairly simple. We shall indicate how the space $H^{\infty}$ of all bounded analytic functions in $|z|<1$ may be regarded as a Banach algebra, and then introduce the space $\mathfrak{S}$ of all complex homomorphisms of $H^{\infty}$, or the maximal-ideal space of $H^{\infty}$. The Shilov boundary will be introduced as the smallest closed subset of $\mathfrak{H}$ which satisfies an Iversen-type property.

It will be convenient to adopt certain minor changes of notation. Thus, let $H^{\infty}$ denote the space of all bounded analytic functions in the open unit disc $D=\{\lambda|| \lambda \mid<1\}$. Then $H^{\infty}$ becomes a Banach algebra with identity under pointwise operations and under the supremum norm

$$
\|f\|=\sup _{\lambda \in D}|f(\lambda)| .
$$

We denote by $\mathfrak{H}$ the space of all complex homomorphisms of the algebra $H^{\infty}$; the elements of $\mathfrak{S}$ are complex homomorphisms of $H^{\infty}$ into the algebra of complex numbers, that is, the elements of $\mathfrak{H}$ are the multiplicative linear functionals on $H^{\infty}$; for each point $\varphi$ in $\mathfrak{S}$ the kernel of $\varphi$ is a maximal ideal in the algebra $H^{\infty}$, and conversely every maximal ideal in $H^{\infty}$ arises in this way. It is also known that $\mathfrak{S}$ is contained in the unit sphere of the conjugate space $\left(H^{\infty}\right)^{*}$ of $H^{\infty}$. Since the unit sphere 
in $\left(H^{\infty}\right)^{*}$ is weak-star compact, it follows that $\mathfrak{H}$ is a compact Hausdorff space when it is endowed with the weak-star topology. A basic weak-star neighbourhood of a point $\varphi_{0}$ in $\mathfrak{S}$ has the form

$$
U\left(f_{1}, \ldots, f_{n}, \varphi_{0}, \varepsilon\right)=\left\{\varphi \in \mathfrak{H}|| \varphi\left(f_{i}\right)-\varphi_{0}\left(f_{i}\right) \mid<\varepsilon ; i=1, \ldots, n\right\},
$$

where $f_{1}, \ldots, f_{n}$ is any finite set of elements in $H^{\infty}$, and where $\varepsilon>0$.

With each function $f$ in $H^{\infty}$ we associate a complex-valued function $\hat{f}$ on $\mathfrak{H}$, where $\hat{f}$ is defined by $\hat{f}(\varphi)=\varphi(f)$. By the definition of the weakstar topology on $\mathfrak{H}$, each $\hat{f}$ is continuous on $\mathfrak{H}$. If $\hat{H}^{\infty}$ denotes the set of all functions $\hat{f}$, then the mapping $f \rightarrow \hat{f}$ is an algebra isomorphism of $H^{\infty}$ onto $\hat{H}^{\infty}$, which is usually called the Gelfand representation. In the case of the Banach algebra $H^{\infty}$, the Gelfand representation is also isometric, that is, $\sup _{\varphi \in \mathfrak{S}}|\hat{f}(\varphi)|=\|f\|$. The complex homomorphisms of $H^{\infty}$, which are easy to identify, are those which arise from points in the open unit disc, namely, $\varphi_{\lambda}(f)=f(\lambda)$. There is a natural projection map $\pi$ of $\mathfrak{H}$ onto $|\lambda| \leqq 1$, which is defined by $\pi(\varphi)=\varphi(z)$ for all $\varphi$ in $\mathfrak{S c}$; in other words, $\pi$ is the mapping $\hat{z}$. The following result was proved by Schark [22].

$\mathrm{Th}$ e o r e m 8. The projection $\pi$ is a continuous map of $\mathfrak{H}$ onto the closed unit disc in the plane. If $\Delta=\pi^{-1}(D)$, then $\pi$ maps the open set $\Delta$ homeomorphically onto the open disc $D$.

Since the set $\Delta$ of all point-evaluation homomorphisms is an open set in $\mathfrak{S}$, the remainder of $\mathfrak{\mathscr { C }}$, namely $\mathfrak{H}-\Delta$, is mapped by $\pi$ onto the circle $\mathfrak{C}=\{\alpha|| \alpha \mid=1\}$. If $\alpha$ is a point of $\mathfrak{S}$, we shall call $\pi^{-1}(\alpha)$ the fiber of $\mathfrak{\mathscr { C }}$ over $\alpha$, and we shall denote this fiber by $\mathfrak{H}_{\alpha}$,

$$
\mathfrak{H}_{\alpha}=\pi^{-1}(\alpha)=\{\varphi \in \mathfrak{H} \mid \varphi(z)=\alpha\} .
$$

The fundamental connection between the cluster set $C(f, \alpha)$ of a function $f \in H^{\infty}$ at $\alpha \in \mathcal{C}$ and the maximal-ideal space is given by the following result of Schark [22].

Th e o r e m 9. Let $f$ be a function in $H^{\infty}$, and let $\alpha$ be a point on $C$. Then $\hat{f}\left(\mathfrak{H}_{\alpha}\right)=C(f, \alpha)$.

We define the Shilov boundary $\Gamma$ as the smallest closed subset of $\mathfrak{H}$ such that every function in $\hat{H}^{\infty}$ attains its maximum modulus at some point of $T$,

$$
\sup _{\varphi \in T}|\hat{f(\varphi)}|=\sup _{\varphi \in \mathfrak{H}}|\hat{f}(\varphi)| .
$$

We know that, in the maximal-ideal space $\mathfrak{H}$ of $H^{\infty}$, the Shilov boundary $\Gamma$ exists and is unique, and, by the maximum-modulus principle, $\Gamma$ is contained in $\mathfrak{S}-\Delta$. A point $\varphi_{0}$ belongs to $\Gamma$ if and only if, for every neighbourhood $N\left(\varphi_{0}\right)$ of $\varphi_{0}$, there exists a function $\hat{g}$ in $\hat{H}^{\infty}$ whose 
absolute value assumes its maximum in $N\left(\varphi_{0}\right)$ and is less than this maximum outside $N\left(\varphi_{0}\right)$.

Finally, if we denote by $\hat{H}_{\alpha}^{\infty}$ the algebra obtained by restricting $\hat{H}^{\infty}$ to the fiber $\mathfrak{H}_{\alpha}$, we have (Hoffman [12]) that (i) $\hat{H}_{\alpha}^{\infty}$ is a uniformly closed subalgebra of $C\left(\mathfrak{H}_{\alpha}\right)$, the algebra of all continuous complex-valued functions on $\mathfrak{H}_{\alpha}$; (ii) the maximal-ideal space of $\hat{H}_{\alpha}^{\infty}$ is $\mathfrak{S}_{\alpha}$; (iii) the Shilov boundary of $\hat{H}_{\alpha}^{\infty}$ is $\Gamma_{\alpha}=\Gamma \cap \mathfrak{H}_{\alpha}$. Furthermore, each fiber is connected and $\mathfrak{\mathfrak { Y }}-\Delta=\mathrm{U}_{\alpha \in \mathfrak{C}} \mathfrak{\mathscr { S }}_{\alpha}$ is connected.

It is known in the general theory of Banach algebras that the image of any function on the Shilov boundary contains the frontier of the image of the function on the maximal-ideal space. In the case of the algebra $\hat{H}_{\alpha}^{\infty}$, this result takes the following form (Max Weiss [23]): For any point $\alpha$ on $|z|=1$ and for any function $\hat{f}$ in $\hat{H}_{\alpha}^{\infty}, \quad \partial \hat{f}\left(\mathfrak{H}_{\alpha}\right) \subseteq \hat{f}\left(\Gamma_{\alpha}\right)$.

We shall say that a closed subset $s$ of $\mathfrak{S}$ has the Iversen property if, for every function $\hat{f}$ in $\hat{H}^{\infty}, \partial \hat{f}(\mathfrak{H}-\Delta) \subseteq \hat{f}(s)$. The Shilov boundary $\Gamma$ $i s$ then the smallest closed subset of $\mathfrak{H}$ which has the Iversen property. Both Schark [22] and Hoffman [12] found the topology on $\mathfrak{H}-\Delta$ to be extremely curious in this sense: In any given fiber $\mathfrak{H}_{\alpha}$ some of the points are limit points of points in fibers $\mathfrak{H}_{\beta}$ with $\beta$ close to $\alpha$, on one side of $\alpha$ or the other, whereas some points of $\mathfrak{H}_{\alpha}$ cannot be approached by points from any other fibers $\mathfrak{\mathfrak { C }}_{\beta}$. It was Max Weiss [23] who first pointed out the connection between this behaviour and the classical theorem of Iversen (Theorem 3 above), and who made a deep investigation of the significance of theorems of Gross-Iversen type in the algebra of bounded analytic functions.

According to Weiss, an operator $K$ in $H^{\infty} \times \mathcal{C}$ to subsets of the plane is called a cluster operator if, for each $f$ in $H^{\infty}$ and $\alpha$ in $c, K(f, \alpha)$ is a subset of the cluster set $C(f, \alpha)$ of $f(z)$ at $\alpha$. If $K$ is a cluster operator, the set $K(f, \alpha)$ is a cluster set of $f(z)$ at $\alpha$. The set of all cluster operators may be partially ordered, and we write $K \prec K^{\prime}$ if $K(f, \alpha) \subset K^{\prime}(f, \alpha)$ for each $f(z)$ and $\alpha$. In particular, the sets $C(f, \alpha), C_{B}(f, \alpha)$, and $C_{R-E}(f, \alpha)$ defined in Sections 1 and 2 give rise to the cluster operators $C, C_{B}$, and $C_{R-E}$. The definition also includes the operator $R$ whose value at $(f, \alpha)$ is the range $R(f, \alpha)$, as well as the operator $\partial C$ whose value at $(f, \alpha)$ is the frontier $\partial C(f, \alpha)$ of $C(f, \alpha)$. Now by Theorem 9 , the cluster set $C(f, \alpha)$ is the image under $\hat{f}$ of the fiber $\mathfrak{H}_{\alpha}$ over $\alpha$, and similarly the radial cluster set $C_{\text {rad }}(f, \alpha)$ is the image under $\hat{f}$ of a compact subset of $\mathfrak{S}_{\alpha}$ : the compact subset is the intersection of $\mathfrak{H}_{\alpha}$ with the closure in $\mathfrak{S}$ of the radius drawn to $\alpha$. A cluster operator $K$ is called a uniform cluster operator if for each $\alpha \in \mathfrak{C}$ there is a closed subset 
$K_{\alpha}$ of $\mathfrak{H}_{\alpha}$ such that, for each function $f(z)$ in $H^{\infty}, K(f, \alpha)=\hat{f}\left(K_{\alpha}\right)$. The analogues of the sets (2) and (5) are obtained in the following way. Let $J$ be any collection of subsets of $\sqrt{ }$ which contains the empty set and which is closed under finite unions. For each $\alpha \in \mathfrak{C}$ let $J(\alpha)$ be the collection of all differences, where $N$ is a neighbourhood of $\alpha$, where $M \in J$, and where $\alpha \in N-M$. Let $K$ be a given cluster operator and define the operator $K^{J}$ for each $f$ in $H^{\infty}$ and $\alpha$ on $\subseteq$ by

$$
K^{J}(f, \alpha)=\bigcap_{N \in J(\alpha)}\left\{\bigcup_{\beta \in N-\{\alpha\}}^{U} K(f, \beta)\right\} .
$$

It is clear that $K^{J}$ is a cluster operator and that $K^{J}(f, \alpha)$ is a subset of $C_{B}(f, \alpha)$. The first main result of Weiss [23] is that $K^{J}$ is a uniform cluster operator whenever $K$ is. All the cluster operators mentioned above are uniform cluster operators with the exception of $R$ and $\partial C$.

The most important of the cluster operators considered by Weiss was the Shilov boundary cluster operator $S$ defined by $S(f, \alpha)=\hat{f}\left(\Gamma_{\alpha}\right)$, that is, $S(f, \alpha)$ is the image under $\hat{f}$ of that part of the Shilov boundary of $\mathfrak{H}$ in the fiber $\mathfrak{S}_{\alpha}$. The following result relates the Shilov operator to the results in Section 2.

The or em 10. For each $f$ in $H^{\infty}$ and $\alpha$ on $\mathbb{C}$,

$$
S(f, \alpha)=\bigcap_{E \in J_{m}} C_{R-E}(f, \alpha),
$$

where the symbolism $E \in J_{m}$ means that $E$ ranges over all sets of Lebesgue measure zero on $\mathfrak{C}$.

Theorem 10 also shows that for each $f$ in $H^{\infty}$ and $\alpha$ on $C$ there exists a set $E$ on $C$ of measure zero such that $S(f, \alpha)=C_{R-E}(f, \alpha)$. In terms of Picard-type behaviour, Theorem 10 leads to the following result, which is a special case of Theorem 6 above.

$\mathrm{Th}$ e o r e m 11. Let $f$ be a function in $H^{\infty}$ and $\alpha$ a point of $\mathbb{C}$. Then the set

$$
\begin{aligned}
& C(f, \alpha)-S(f, \alpha) \text { is open and the set } \\
& C(f, \alpha)-S(f, \alpha)-R(f, \alpha) \text { is of capacity zero. }
\end{aligned}
$$

It should be remarked that, even though the classical results (such as Theorem 6) were proved for meromorphic functions, the restriction to functions of $H^{\infty}$ does not involve a real loss of generality, because the proofs in the more general case involved a localization principle in which a reduction to bounded analytic functions was made ([18], Chapter 4).

We remark that there are some immediate corollaries of the above results. First, it has been pointed out by Csordás [3] that if $C_{\operatorname{rad}}(f, \alpha)$ is the radial cluster set of $f(z)$ at $\alpha$, then the set of points $\alpha$ on $|z|=1$ 
such that $C_{\text {rad }}(f, \alpha) \cap \hat{f}\left(\Gamma_{\alpha}\right)$ is empty is of measure zero. Furthermore, a necessary and sufficient condition that a function $f(z)$ in $H^{\infty}$ be an inner function is that the image of the Shilov boundary be a subset of $|z|=1$ (Csordás [3]; see also Hoffman [12], who showed that $|\hat{f}(\Gamma)|=1$ if $f$ is an inner function).

4. Gleason Parts. Despite the fact that each fiber $\mathfrak{H}_{\alpha}$ has no interior, it is known [22] that $\mathfrak{H}_{\alpha}$ contains a homeomorphic image of $\mathfrak{\mathfrak { H }}$. A further study of the fibers $\mathfrak{S}_{\alpha}$ may be carried out by introducing equivalence classes of complex homomorphisms called Gleason parts by using the pseudo-hyperbolic distance

$$
\chi\left(z_{1}, z_{2}\right)=\left|\frac{z_{1}-z_{2}}{1-\bar{z}_{2} z_{1}}\right|, \quad\left|z_{1}\right|<1, \quad\left|z_{2}\right|<1,
$$

which is invariant under conformal mappings of $|z|<1$ onto $|z|<1$. We now define the pseudo-hyperbolic distance on $\mathfrak{S}$ by

$$
\chi\left(\varphi_{1}, \varphi_{2}\right)=\sup _{\|f\|<1} \chi\left(\varphi_{1}(f), \varphi_{2}(f)\right)
$$

and we define $\varphi_{1}$ and $\varphi_{2}$ to be equivalent if $\chi\left(\varphi_{1}, \varphi_{2}\right)<1$. The equivalence classes into which $\mathfrak{S}$ is partitioned are called Gleason parts. The motivation for introducing the Gleason parts comes from the analytic structure of certain subsets of $\mathfrak{H}$. A map $\psi$ from $D:|z|<1$ into $\mathfrak{H}$ is called analytic if, for each $f$ in $H^{\infty}$, the map $\hat{f} \circ \psi$ is analytic on $D$. Let $P(\varphi)$ be the Gleason part to which $\varphi$ belongs; if $\psi$ is analytic and if $\psi(\lambda)=\varphi$ for some $\lambda \in D$, then $\psi(D) \subseteq P(\varphi)$. Hoffman [13] established many facts concerning the relations between Gleason parts and interpolating sequences; we use the terminology that if $\left\{\lambda_{n}\right\}$ is an interpolating sequence in $D$, then $\left\{\varphi_{\lambda_{n}}\right\}$ is an interpolating sequence in $\Delta$. Then each point of the image of $D$ under a non-constant analytic mapping lies in the closure of an interpolating sequence.

Th e or e m 12 (Hoffman [13]; Schark [22]). The transformation from $\triangle$ into $\mathfrak{S C}^{D}$, the space of all maps from $D$ into $\mathfrak{H}$, which assigns to the evaluation homomorphism $\varphi_{\lambda}=\pi^{-1}(\lambda)$ the linear fractional map $\Lambda_{\lambda}=$ $\pi^{-1}\left(L_{\lambda}\right)$, where

$$
L_{\lambda}(z)=\frac{z+\lambda}{1+\bar{\lambda} z}
$$

can be extended uniquely to a continuous transformation from $\mathfrak{H}$ into $\mathfrak{S C}^{D}$. If $\varphi$ is a point of $\mathfrak{S}$ which does not lie in the closure of an interpolating sequence, then the corresponding map $\Lambda_{\varphi}$ is constant, while if $\varphi$ lies in the closure of some interpolating sequence, then $\Lambda_{\varphi}$ is a one-to-one analytic map of $D$ onto the Gleason part $P(\varphi)$. 
We remark that if $\varphi$ is a point for which $\Lambda_{\varphi}$ is constant, then $P(\varphi)$ reduces to the single point $\varphi$. Now it is known that the pseudo-hyperbolic distance $\chi$ is lower semicontinuous, but not continuous, on $\mathfrak{H} \times \mathfrak{H}$ and that the connected components of the metric space $(\mathfrak{S}, \chi)$ are the Gleason parts in $\mathfrak{S}$. There exists [22] an analytic disc in $\mathfrak{H}-\Delta$, so that there exist non-trivial parts besides the disc $\Delta$, and Kerr-Lawson [17] showed that non-tangential and oricyclic points in $\mathfrak{S}$ possess non-trivial parts. A point $\varphi$ in the fiber $\mathfrak{H}_{\alpha}$ is called a non-tangential point or a Stolz point if it lies in the closure of a sector bounded by two line segments in $D$ with common endpoint $\alpha$. A point is called oricyclic if it lies in the closure of the region between two oricycles tangent to $|z|=1$ at $\alpha$. Every oricyclic point, as well as every Stolz point, of $\mathfrak{H}$ lies in the closure of an interpolating sequence.

We conclude with an extension of the notion of tangential limit. Ebert [7] investigated the endomorphisms of $H^{\infty}$; these were related to their adjoints, which constitute a special class of mappings of $\mathfrak{H}$ into $\mathfrak{H}$. As an application of the structure of the space of endomorphisms, it was shown that each analytic part in a fiber has an order of tangency, that is, a number which provides a partial description of any subset of $\Delta$ whose closure intersects the given analytic part. Let $G$ be a set in $D$ which contains in its closure the point $\alpha$. We define the upper and lower orders of tangency of $G$ at $\alpha$ to be

$$
\begin{aligned}
& \bar{t}(G)=\lim \sup \frac{\log (1-|\zeta|)}{\log |\alpha-\zeta|}-1, \\
& \underline{t(G)}=\liminf \frac{\log (1-|\zeta|)}{\log |\alpha-\zeta|}-1
\end{aligned}
$$

where $\zeta$ tends to $\alpha$ through the set $G$. If $\bar{t}(G)=t(G)$, the common value is called the order of tangency of $G$ and is denoted by $t(G)$. These numbers may be any non-negative real numbers, or infinity. For example, a Stolz path ending at $\alpha$ has order of tangency zero, and an oricycle of radius $R$ tangent to $|z|=1$ at $\alpha$ has order of tangency 1 , independent of the radius $R$. The main result of Ebert in this direction is the following.

$\mathrm{Th}$ e o r e m 13. If $\varphi$ belongs to an analytic part, then $\varphi$ has a unique order of tangency, and two points belonging to the same analytic part have the same orders of tangency.

We remark that, for each $n, 0 \leqq n \leqq \infty$, it is not difficult to construct an interpolating sequence with order of tangency $n$ at $z=1$. Moreover, the closure of the sequence in $\mathfrak{S}$ contains only points lying in analytic parts. 


\section{References}

[1] CARTWRIGHT, M. L.: The exceptional values of functions with a non-enumerable set of essential singularities. - Quart. J. Math. Oxford Ser. 8, 1937, $303-307$.

[2] Colingwood, E. F., and A. J. Lohwater: The theory of cluster sets. - Cambridge Tracts in Mathematics and Mathematical Physics 56. University Press, Cambridge, 1966.

[3] Csordás, G. L.: The Silov boundary and a class of functions in $H^{\infty}$. - Ph. D. Dissertation, Case Western Reserve University, Cleveland, Ohio, 1969.

[4] Doob, J. L.: On a theorem of Gross and Iversen. - Ann. of Math. (2) 33, 1932, 753-757.

[5] -»- One-sided cluster-value theorems. - Proc. London Math. Soc. (3) 13, 1963, 461-470.

[6] Duren, P. L.: Theory of $H^{p}$ spaces. - Pure and Applied Mathematics 38. Academic Press, New York-London, 1970.

[7] EBER', W. L.: Endomorphisms, orders of tangency, and applications to $H^{\infty}$. Ph. D. Dissertation, Case Western Reserve University, Cleveland, Ohio, 1969.

[8] GEL'FAND, I. M., D. A. RA1̆Kov and G. E. ŠILOV [И. М. Гельфанд, Д. А. Райков and Г. Е. Шилов]: Коммутативные нормированные кольца. [Commutative normed rings.] - Uspehi Mat. Nauk (N.S.) 1:2 (12), 1946, 48-146.

[9] Gross, W.: Über die Singularitäten analytischer Funktionen. - Monatsh. Math. Phys. 29, 1918, 3-47.

[10] -»- Zum Verhalten analytischer Funktionen in der Umgebung singulärer Stellen. - Math. Z. 2, 1918, 242-294.

[11] AF HÄLLSTRÖM, G.: Über eindeutige analytische Funktionen mit unendlich vielen wesentlichen Singularitäten. - Neuvième congrès des mathématiciens scandinaves à Helsingfors du 23 au 26 août 1938, Helsinki, $1939,277-284$.

[12] Hoffman, K.: Banach spaces of analytic functions. - Prentice-Hall, Inc., Englewood Cliffs, New Jersey, 1962.

[13] -»- Bounded analytic functions and Gleason parts. - Ann. of Math. (2) 86, 1967, 74-111.

[14] IVERSEN, F.: Recherches sur les fonctions inverses des fonctions méromorphes. Thèse présentée à la Faculté des Sciences de l'Université de Helsingfors, 1914.

[15] -»- Sur quelques propriétés des fonctions monogènes au voisinage d'un point singulier. - Öfversigt af Finska vetenskaps-societetens förhandlingar 58.A.25, Helsingfors, 1915-1916, 1-16.

[16] - - Zum Verhalten analytischer Funktionen in Bereichen, deren Rand eine wesentliche Singularität enthält. - Översikt av Finska vetenskapssocietetens förhandlingar 64.A.4. Helsingfors, 1921-1922, 1-19.

[17] KERR-LAwSON, A.: A filter description of the homomorphisms of $H^{\infty}$. Canad. J. Math. 17, 1965, 734-757.

[18] LoHwater, A. J. [A. Ловатер]: Граничное Поведение аналитических функций. [The boundary behaviour of analytic functions]. - Izdat. Viniti, Moscow, 1973.

[19] Natmark, M. A.: Normed rings. - P. Noordhoff N. V., Groningen, 1959.

[20] RICKART, C. E.: General theory of Banach algebras. - D. Van Nostrand 
Company, Inc., Princeton, New Jersey - Toronto-London-New York, 1960.

[21] Rudin, W.: Function theory in polydiscs. - W. A. Benjamin, Inc., New York1. Amsterdam, 1969.

[22] Schark, I. J.: Maximal ideals in an algebra of bounded analytic functions. J. Math. Mech. 10, 1961, 735-746.

[23] WEIsS, M. L.: Cluster sets of bounded analytic functions from a Banach algebraic viewpoint. - Ann. Acad. Sci. Fenn. Ser. A I 367, 1965, 1-14.

[24] WERMER, J.: Banach algebras and analytic functions. - Advances in Math. 1, $1965[1961], 51-102$.

[25] WOOLF, W. B.: Radial cluster sets and the distribution of values of meromorphic functions. - Ph. D. Dissertation, University of Michigan, Ann Arbor, 1959.

[26] - - The boundary behavior of meromorphic functions. - Ann. Acad. Sci. Fenn. Ser. A I 305, 1961, 1-11.

\author{
Case Western Reserve University \\ Department of Mathematics \\ Cleveland, Ohio 44106 \\ USA
}

Received 30 October 1975 